\title{
Turismo alternativo y localización territorial: el caso de la Península de Yucatán, México
}

\section{Ana García de Fuentes* María Dolores Cervera Montejano** \\ CINVESTAV Unidad Mérida (México)}

\begin{abstract}
Resumen: El impulso oficial al turismo alternativo busca la conservación ambiental y el mejoramiento de las condiciones de vida de poblaciones indígenas y campesinas con resultados relativos. Considerando que la localización es central al desempeño económico, medido por número de turistas e ingresos, analizamos estadísticamente sus relaciones con tres tipos de localización, además del financiamiento total. Utilizamos información del Atlas de Turismo Alternativo de la Península de Yucatán. Los resultados muestran que los ingresos dependen del número de turistas, pero la ubicación puede modificar esta asociación. El mejor desempeño económico se relaciona con la cercanía a lugares de turismo masivo. Sin embargo, la política de fomento al turismo alternativo parece basarse en la idea de que todos los lugares pueden convertirse en productos turísticos sin considerar su localización territorial.
\end{abstract}

Palabras Clave: Turismo alternativo; Empresas sociales; Localización territorial; Desempeño económico; Yucatán.

\section{Alternative tourism and territorial location: the case of the Yucatán Península, México}

Abstract: The official promotion of alternative tourism seeks environmental conservation and the improvement of the living conditions of indigenous and peasant populations with relative success in the results thus far. Considering that location is central to economic performance, measured by number of tourists and income plus total financing, we analysed statistically alternative tourism's results over three types of location. We used information from the Alternative Tourism Atlas of the Yucatan Peninsula. The results show that income depend on the number of tourists, but that location may alter this link. The best economic performance is given in the location close to mass tourism sites. However, the policy of promoting alternative tourism seems to be based on the idea that all places can become tourist products without considering their territorial location.

Keywords: Alternative tourism; Social enterprises; Territorial location; Economic performance; Yucatan.

\section{Introducción}

El turismo alternativo es una modalidad del turismo que surge en la década de 1970 y adquiere gran importancia a partir de la última década del siglo XX, período en que alcanza tasas de crecimiento anual de $20 \%$ a nivel mundial, convirtiéndose en el segmento del sector turístico con mayor crecimiento (OMT, 2001).

El término alternativo se aplica, en relación al turismo, en al menos dos acepciones que en la práctica operan como complementarias:

1) La primera, de carácter ambientalista, se planteó en la Conferencia Mundial de Turismo Sostenible realizada en Lanzarote en 1995, como resultado de los debates sobre sostenibilidad surgidos en la Cumbre de la Tierra en Río de Janeiro en 1992. Propone desarrollar un turismo alternativo al modelo masivo de sol y playa, con prácticas más amigables con el entorno y una gestión que garantice la perdurabilidad de los recursos naturales (OMT, 1995). Esta acepción, ampliamente

* Dpto. Ecología Humana. CINVESTAV - Unidad Mérida (México); E-mail: agarcia@cinvestav.mx

** Dpto. Ecología Humana. CINVESTAV - Unidad Mérida (México); E-mail: dolores.cervera@cinvestav.mx 
difundida, se vincula con lo que plantea Cheia (2013) de que el turismo se reinventa a través de un consumo más emocional basado en la venta de experiencias únicas, vividas en los lugares que se visitan; se vende así el contexto tanto natural como cultural promoviendo un consumo responsable y amigable con el entorno.

2) Una segunda acepción es la del turismo como una alternativa a la agricultura tradicional, abandonada en México por parte de las políticas públicas. Se plantea como una forma de que los campesinos logren mejorar sus condiciones de vida, permaneciendo en sus lugares de origen. En la práctica, con pocas excepciones, más que una alternativa a la agricultura opera como una opción complementaria que se inserta en la compleja diversidad productiva que permite la sobrevivencia de estos campesinos (agricultura, recolección, cacería, producción artesanal, migración temporal, y ahora oferta turística).

Los dos planteamientos convergen en su interés por incorporar nuevos territorios al turismo. Estos territorios se caracterizan por tener una gran riqueza biocultural, y por ubicarse en regiones marginales al desarrollo capitalista, como son los territorios indígenas, donde se ofrece a grupos selectos el contacto con la naturaleza "prístina" y con la "auténtica" cultura local.

El impulso para que los campesinos participen en este tipo de turismo responde a una estrategia del Estado y de organismos internacionales que, mediante financiamientos a fondo perdido - es decir, subsidios - promueven la creación de empresas sociales dedicadas al turismo en las comunidades rurales, las que se insertan en sistemas mercantiles en los que operan con fuertes desventajas competitivas (CONEVAL s/f; Coraggio, 2011; García de Fuentes, Jouault, y Romero, 2015; Jiménez, 2016; Marañón y López, 2013; Pulido 2014).

Diversos autores defienden las bondades ambientales y económicas de este tipo de turismo (Ceballos, 1998; Salciccia, 2001; Garduño et al., 2009; Félix-Quezada; 2016); sin embargo, otros plantean que en la práctica dista mucho de cumplir con los objetivos de sostenibilidad (Bringas, y Ojeda, 2000; Daltabuit, et al., 2007). Barragán (2015) señala que la conservación pasiva que practican la mayoría de estas empresas tiene impactos muy limitados; reconoce, sin embargo, el papel de conservación activa de algunos grupos, en particular en la costa norte de Yucatán.

Desde el punto de vista económico, Gasca et al. (2010) señalan las múltiples dificultades que enfrentan estas empresas para ser competitivas en términos de comercialización, difusión y promoción de la oferta y, sobre todo la gran dificultad de conciliar la lógica empresarial con las formas de organización y producción campesinas. En el mismo sentido Jouault, García de Fuentes y Rivera (2015) destacan los numerosos fracasos, expresados en el abandono de los proyectos a los pocos años.

Por su parte, autores como Fletcher (2016), López, A. (2015) y López, A. y Marín (2012) consideran que este tipo de turismo es una forma sofisticada del capital para apropiarse de territorios biodiversos y con un capital cultural que, generalmente, se encuentran en manos de los pueblos indígenas. Se trata de aprovechar el incremento brutal de los flujos de turistas, para lo cual, como señala Urry (2001), se reconfiguran cada vez más lugares como sitios de producción y consumo global del turismo. En esta construcción social, donde interviene la población local, pero también agentes externos y particularmente los distintos órdenes de gobierno, pareciera que todos los lugares aspiran a ser productos turísticos.

En México los únicos estudios de carácter cuantitativo sobre este tipo de turismo son los publicados por López, G. y Palomino en 2008 y 2015, en los que presentan un análisis a escala nacional de las empresas indígenas de turismo de naturaleza, a partir de información de fuentes oficiales como SECTUR y la CDI, entre otras, y de páginas web de las empresas. Desde el punto de vista de los resultados económicos atribuyen el éxito en estas empresas al uso de Tics, páginas web y a los apoyos de financiamiento recibidos, si bien también mencionan que la integración a rutas turísticas o a paquetes estructurados y las vías de acceso inciden en el éxito de estos negocios. En un trabajo posterior presentan una posición más crítica señalando la gran heterogeneidad de los proyectos que obedece a factores internos como las formas de organización colectiva, y a los contextos externos en que se desarrollan (Palomino et. al, 2016).

En la península de Yucatán, el Atlas de Turismo Alternativo en la Península de Yucatán (García de Fuentes et al., 2015) reporta resultados de un amplio estudio censal al total de las 153 empresas colectivas (cooperativas, ejidales, registradas bajo otras modalidades y no registradas), existentes en 2013 y ubicadas en el medio rural. En la Península, el Programa Mundo Maya, que empezó a gestarse en 1988, marcó el inicio de la promoción del turismo en los espacios rurales y, en la década del 2000, la iniciativa de la OMT permeó los esquemas de promoción turística de México, incorporando a ellos el impulso al turismo alternativo. En este escenario y con el apoyo de instituciones gubernamentales y civiles nacionales e internacionales, varias localidades rurales localizadas tanto en el interior como en 
el litoral del territorio peninsular se convierten en una oferta de turismo alternativa complementaria a la del turismo clásico (García de Fuentes et al., 2015).

El análisis espacial del Atlas hizo evidentes diversos procesos que plantean nuevas interrogantes sobre la forma en que se desenvuelve este tipo de turismo. Entre ellos, mostró que la localización es un factor clave para el éxito de ese tipo de empresas. Con estos antecedentes, en este artículo utilizamos la información del Atlas para analizar estadísticamente las relaciones entre la localización territorial de las empresas, como factor clave para su desempeño económico, medido a través de dos indicadores: el número anual de turistas atendidos y el ingreso bruto anual. Agregamos también el financiamiento total acumulado en el período de 1994 a 2012. Partimos de la consideración de que existe una relación entre ubicación y desempeño económico, así como que la ubicación no es criterio para otorgar el financiamiento.

\section{Hipótesis}

La localización territorial de las empresas desempeña un papel fundamental en términos de su factibilidad económica y pervivencia social, es decir, la localización sí importa.

Preguntas de investigación:

1) ¿Existen diferencias significativas entre la ubicación de las empresas y el número de turistas recibidos?

2) ¿Existen diferencias significativas entre la ubicación de las empresas y el ingreso bruto anual percibido?

3) ¿Existen diferencias significativas entre la ubicación de las empresas y el monto del financiamiento acumulado?

\section{La multidimensionalidad de la localización territorial}

A partir de la información del Atlas se identificaron tres tipos de ubicación que muestran diferencias desde el proceso de creación de las empresas del sector social hasta su desempeño: 1 . Diferencias entre el litoral y los territorios del interior; 2. Diferencias entre el litoral del Caribe frente al litoral de Yucatán y Campeche y 3. Diferencias entre el traspaís de Cancún-Riviera Maya y el resto de los territorios interiores (ver Figura 1).

Del total de 153 empresas estudiadas, 88 (57.5\%) se ubican en el litoral y 65 (42.5\%) en el interior. De las 25 empresas más antiguas, 21 se ubican en el litoral. Éstas, al igual que otras de surgimiento posterior, se organizaron de manera endógena como respuesta a una demanda del mercado turístico (internacional y nacional en el caso del Caribe y local en Yucatán, costa de Campeche y sur de Quintana Roo) que al visitar la zona buscó a los pescadores locales para contratar paseos en lancha y transporte para snorquelear. Además, en la zona del Caribe, los pescadores empezaron a complementar la oferta con alimentos del mar durante el recorrido. Este antecedente nos permite afirmar que la incorporación, y en ocasiones reconversión total, de los pescadores a turisteros en el litoral de la península, al igual que en la mayor parte de los centros turísticos de sol y playa, se origina de manera espontánea en respuesta a una demanda in situ.

Vinculado a lo anterior, la constitución de cooperativas de turismo en el litoral tiene como antecedente que la mayoría de sus socios pertenecieron o siguen perteneciendo a una cooperativa pesquera, heredando con ello cierta cultura cooperativa. En el caso de México, esta cultura ha estado fuertemente vinculada a las directrices del Estado y a la dependencia de los permisionarios, lo que impide el desarrollo de una organización verdaderamente horizontal (Warman, 2001). En el litoral, 95.5\% de las empresas turísticas del sector social operan bajo la forma de cooperativas. En el interior, éstas también predominan pero sólo representan $53.8 \%$ del total de las empresas, a las que sigue un $29.2 \%$ que opera bajo los esquemas de organización ejidal.

El 90.9\% de las empresas del litoral se ubican en terrenos federales, la mayoría de éstas (83.8\%) cuenta con una concesión federal, si bien en lo general se limita a la zona de instalaciones en la playa de embarque. Los recorridos en lancha se hacen sobre espacios federales de libre acceso, incluyendo playas y manantiales. Por ello, existen pocos conflictos con relación a la tenencia de la tierra, aunque se presentan algunos relacionados con el libre acceso y la basura que deja la población, en particular otros pescadores, en las zonas utilizadas para recreación de los turistas en el litoral. En cambio, en el interior la tenencia de la tierra es más diversa. Del total de empresas, $67.7 \%$ opera en tierras ejidales de uso 


\section{Figura 1: Localización de las empresas turísticas del sector social en la península de Yucatán}

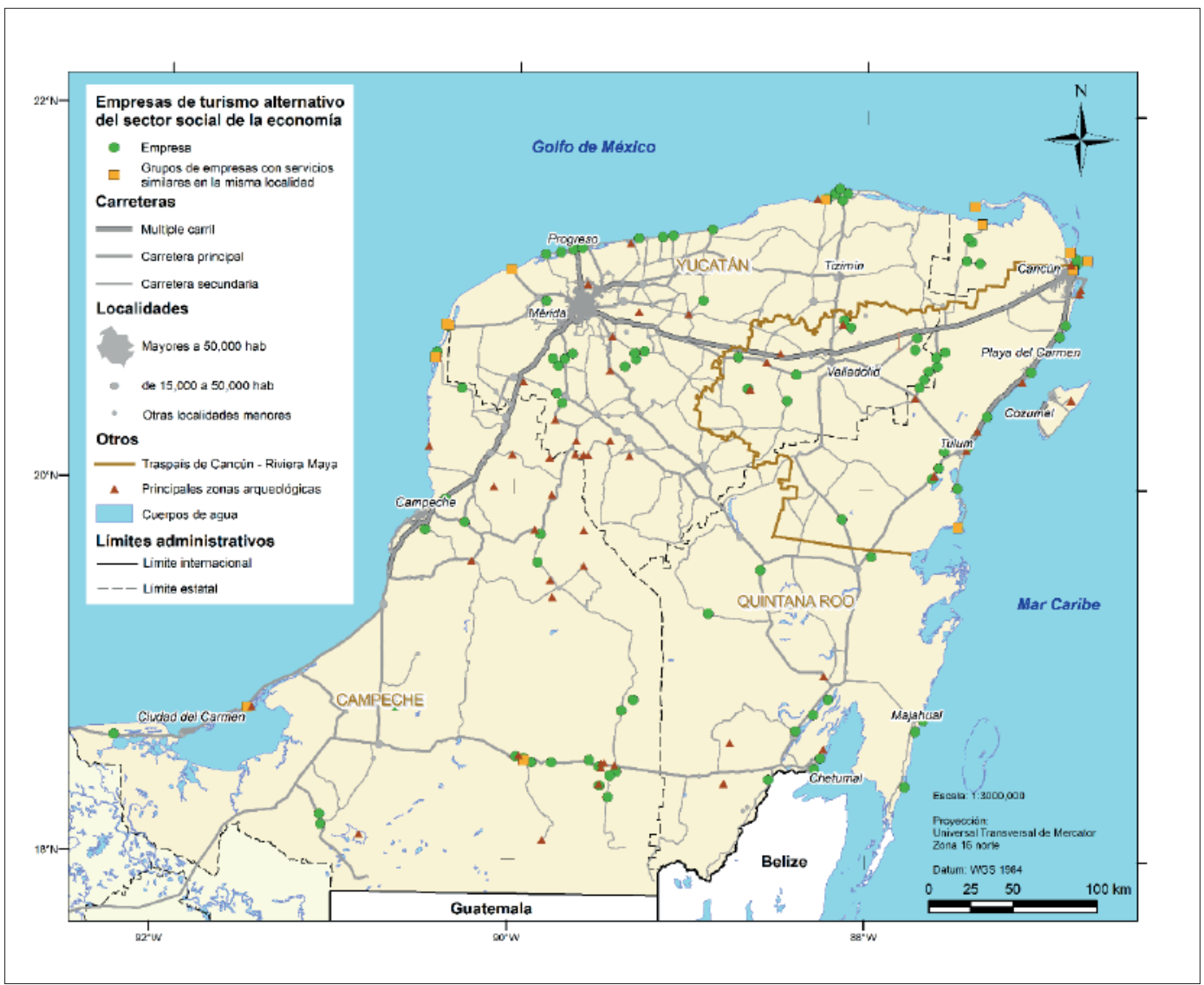

Fuente: Atlas de turismo alternativo en la Península de Yucatán; Secretaria de Fomento Turístico (México).

común, lo que implica acuerdos y, en ocasiones, conflictos entre el grupo que conforma la cooperativa y el resto de los ejidatarios; $12.3 \%$ opera en terrenos ejidales pero ya parcelados y el resto en distintas modalidades que incluyen propiedad privada, renta de tierras y comodatos (13.8\%), sólo 4 empresas $(6.2 \%)$ operan en terrenos federales concesionados.

La intensidad de la actividad también difiere entre ubicaciones, siendo mayor el número de turistas que visitan sitios en el litoral que en el interior. Asimismo, la oferta turística es diferente. En el litoral predominan los paseos en lancha en el mar y en los humedales y manantiales, así como el transporte para bucear o esnorquelear. En el interior destacan los cenotes como un tipo de oferta muy particular de esta región. Sin considerar los privados, en 2013 detectamos 15 cenotes abiertos al turismo, además de que es la oferta de mayor crecimiento, habiéndose duplicado en los últimos años. Esta oferta se complementa con turismo rural y recorridos de naturaleza, así como tirolesas y rappel vinculados a los cenotes.

El ingreso bruto anual y el financiamiento también mostraron ser diferentes. El ingreso fue mayor en el litoral que en el interior. De las 153 empresas, 110 (71.9\%) habían recibido financiamiento, al menos en una ocasión. En este caso, el interior resultó favorecido pues más empresas ubicadas en ese espacio (76.9\%) recibieron financiamiento que empresas ubicadas en el litoral (68.2\%). El financiamiento ha sido otorgado por organismos gubernamentales, siendo los más importantes el Programa de Turismo Alternativo en Zonas Indígenas de la Comisión para el Desarrollo de los Pueblos Indígenas (CDI-PTAZI), la Comisión Nacional de Areas Naturales Protegidas de la Secretaría de Medio Ambiente y Recursos Naturales (CONANP-SEMARNAT) y la Comisión Nacional de Forestal (CONAFOR); organismos 
internacionales, destacando el Programa de Pequeños Donativos del Fondo de Naciones Unidas para el Desarrollo (PPD-PNUD), así como diversas instituciones de la sociedad civil nacional e internacional.

Es importante señalar que el financiamiento otorgado tanto por el Estado mexicano como por organismos internacionales es a fondo perdido - es decir, opera como un susbsidio - y responde a diversas políticas orientadas, por una parte, a contribuir a la conservación de la naturaleza y, por otra, como sustitutos al apoyo a la agricultura tradicional, considerando que el turismo es la alternativa para la sobrevivencia del mundo rural y un medio para frenar la migración campo-ciudad.

Con todo y sus características distintivas, ni el litoral ni el interior son espacios homogéneos. En relación con el litoral, no es lo mismo el del Caribe que el de Yucatán y Campeche. Por sus condiciones naturales y por su relación con el corredor Cancún-Riviera Maya, principal región turística del país y de América Latina, en el primero la oferta de las empresas está ligada al turismo internacional. En el resto de Quintana Roo hay procesos incipientes de desarrollo para el turismo internacional pero su escala es inferior a la del corredor. En el litoral de Yucatán y Campeche, la oferta se relaciona con el turismo de segunda residencia y con la afluencia de visitantes locales, aunque a una escala mucho menor en Campeche. En Yucatán, Celestún puede considerarse una excepción pues es ya destino internacional.

En los territorios interiores destaca la formación del traspaís turístico de Cancún-Riviera Maya, como un triángulo con vértice en Chichen Itzá, que proveé de un mercado a muchas de las empresas del sector, a través de tours organizados desde los principales centros de turismo masivo (Cancún, Playa del Carmen y Tulum). La oferta complementa la estancia del turismo de sol y playa con un tour de unas horas para adentrarse en la naturaleza "tropical", "salvaje" y en la cultura local, es decir "el mundo maya profundo". Mérida crea también un área de influencia en su entorno, relacionada principalmente con la oferta de cenotes. Como contraparte, un conjunto de empresas se ubica en zonas más alejadas, relacionadas sólo con visitantes locales de fin de semana o, en el caso de Calakmul, al sur del estado de Campeche por ejemplo, con una afluencia mínima por su lejanía del mercado.

Lo anterior sugiere que el desempeño de estas empresas se asocia con su ubicación. En otras palabras, la ubicación sí importa en términos de los factores que contribuyen al éxito o fracaso de las empresas sociales dedicadas al turismo. Las ubicadas en el litoral parecen tener un mejor desempeño económico que las empresas del interior. Asimismo, las empresas ubicadas en el litoral Caribe tendrían un mejor desempeño que las del litoral de Yucatán y Campeche, así como las del traspaís sobre las ubicadas en el resto de los territorios interiores de la Península. Sin embargo, el financiamiento parece privilegiar a empresas ubicadas en el interior. Por tanto, el impulso parece ser concebido bajo la premisa de que cualquier lugar puede llegar a ser turístico con solo aspirarlo y un "empujoncito".

\section{Metodología}

El Atlas del Turismo Alternativo en la Península de Yucatán (García de Fuentes, et al., 2015), antecedente básico para la elaboración de este trabajo, es una obra colectiva y multidisciplinaria que, por medio del lenguaje cartográfico, analiza territorialmente las características de este tipo de empresas. Su elaboración se basó en información primaria obtenida directamente en campo mediante la aplicación de un levantamiento censal al total de las empresas del sector inventariadas en la Península de Yucatán. El objetivo fue cubrir la totalidad de las empresas sociales dedicadas al turismo alternativo que estaban en operación en 2013. El cuestionario incluyó 50 preguntas y 180 opciones de respuesta, organizadas en 17 grupos temáticos. El análisis de los mapas y de los cuadros estadísticos descriptivos del Atlas plantea nuevas preguntas de investigación sobre los resultados económicos en la operación de las empresas y su relación con la ubicación geográfica de las mismas, que son las que pretendemos responder en este artículo, a partir del análisis estadístico del comportamiento de tres variables: a) turistas anuales recibidos, b) ingreso bruto anual de las empresas y c) financiamiento total acumulado de 1994 a 2012.

Un problema que enfrentó el Atlas (García et al., 2015) fue la falta de registros administrativos sistemáticos en $53.6 \%$ de las empresas. Para hacer una estimación lo más precisa posible del total anual de turistas y del ingreso bruto anual, se realizó una amplia entrevista a alguno de los representantes de cada empresa, en la que se partió de que la actividad no es continua a lo largo del año y presenta grandes fluctuaciones. De esta manera, se buscó que el entrevistado indicara para el último año, los períodos de afluencia, por ejemplo, fines de semana, solo en verano, Semana Santa, y estimara el número de visitantes en cada caso. También se establecieron los períodos sin visitantes. A partir de este ejercicio, se llegó a un estimado del total de turistas que pudieron haber recibido durante el año. Para calcular el ingreso bruto anual se consideró este número de turistas y las tarifas que aplican, pero 
también se cruzó esta información con los montos de gastos que fueron reportados en otra sección de la misma entrevista. Se optó por utilizar una estimación anual, considerando que era imposible esperar que hubiera ingresos estables durante todo el año, debido a la naturaleza estacional de la actividad. La información sobre el financiamiento total acumulado proporcionada por los entrevistados fue cotejada con los listados de los organismos financiadores, por lo que se puede considerar más precisa. En este caso se optó por sumar todos los financiamientos obtenidos entre 1994 y 2012.

El procesamiento estadístico de la información para este artículo se realizó con el IBM SPSS Statistics, versión 19. El análisis exploratorio de las tres variables mostró que, a pesar de ser continuas, su distribución distaba de acercarse a la normal y que las varianzas eran significativamente diferentes (heteroscedasticidad), aun después de realizar una transformación logarítmica. Por ello, utilizamos estadística no paramétrica. El Cuadro 1 presenta las medidas de tendencia central y de dispersión de las tres variables. El número de empresas ubicadas en el litoral y en el interior fue desigual $(\mathrm{n}=88 \mathrm{y} \mathrm{n}=65$, respectivamente) y la diferencia estuvo cerca de alcanzar significancia estadística $\left(\mathrm{X}_{(1)}^{2}=3.458, \mathrm{p}=0.063\right)$. Es decir, hay una tendencia a un mayor número de empresas en el litoral que en el interior de la península.

Considerando que la ubicación es un factor central del desempeño económico, debería haber una relación positiva entre el número de turistas y los ingresos, es decir a más turistas ingresos mayores, así como una relación positiva del financiamiento tanto con número de turistas como con ingresos. Realizamos un análisis de la relación de las tres variables entre sí mediante diagramas de dispersión y pruebas de correlación de Spearman. Posteriormente, transformamos estas tres variables de continuas a discretas y aplicamos pruebas de $\mathrm{X}^{2}$ para analizar su relación con las tres ubicaciones diferenciadas: litoral/interior, litoral Caribe/litoral Yucatán y Campeche, traspaís/resto del interior. Inicialmente utilizamos las categorías empleadas en el Atlas. Los resultados mostraron celdas con frecuencias esperadas menores a cinco, por lo que redujimos las categorías a tres en cada una de las variables en el caso de las comparaciones entre litoral e interior. En los casos de las comparaciones litoral Caribe/ litoral Yucatán y Campeche y traspaís/resto del interior fue necesario utilizar dos categorías ubicando el punto de corte en la Mediana ( $\leq \mathrm{Md}$, > Md). Para el análisis estadístico de la variable financiamiento total acumulado se incluyeron todas las empresas y no solo aquellas que recibieron apoyo financiero al menos una vez. En este caso, la falta de financiamiento se consideró como financiamiento $\$ 0.00$.

\section{Cuadro 1: Medidas de tendencia central y dispersión de las tres variables seleccionadas para su análisis estadístico $(n=153)$}

\begin{tabular}{|l|r|r|r|}
\hline & \multicolumn{1}{|c|}{ Turistas } & \multicolumn{1}{c|}{ Ingresos } & \multicolumn{1}{c|}{ Financiamiento } \\
\hline Media & $6,890.30$ & $1,494,709.40$ & $972,725.90$ \\
\hline Mediana & $2,810.00$ & $378,000.00$ & $241,709.68$ \\
\hline Moda & $2,000.00$ & $200,000.00^{\mathrm{a}}$ & 0.00 \\
\hline D.E. & $11,054.90$ & $2,293,506.70$ & $1,663,882.04$ \\
\hline Mínimo & 0.00 & 0.00 & 0.00 \\
\hline Máximo & $60,000.00$ & $11,800,000.00$ & $8,315,080.00$ \\
\hline
\end{tabular}

Nota. Turistas $=$ total anual de turistas. Ingresos $=$ ingreso bruto anual. Financiamiento $=$ financiamiento total acumulado 1994-2012.

${ }^{a}$ Se encontraron otras cuatro modas cuyas frecuencias variaron entre 4 ( 0 ingresos) y 2 (ingresos de 9,000.00, $10,000.00,220,000.00$ y 400,000.00).

\section{Resultados}

\section{Relación entre variables}

Los resultados de las pruebas de correlación de Spearman mostraron una relación positiva y estadísticamente significativa entre total anual de turistas e ingresos brutos anuales $(\rho=0.777, p=0.00000)$. A más turistas, más ingresos. No se encontró relación estadísticamente significativa entre turistas y financiamiento $(\rho=-0.072, p=0.379)$ ni entre ingresos y financiamiento $(\rho=-0.107, p=0.186)$. No obstante, es interesante notar que el signo de la relación en ambos casos fue negativo. 


\section{Turistas, ingresos y financiamiento de acuerdo a ubicación en litoral o interior}

Se encontró una asociación positiva y significativa entre ubicación y número de turistas anual $\left(\mathrm{X}^{2}{ }_{(2)}\right.$ $=7.450, \mathrm{p}=0.024)$. Las empresas ubicadas en el interior reciben un número significativamente menor de turistas que las ubicadas en el litoral (Cuadro 2).

Cuadro 2: Comparación del total anual de turistas entre empresas ubicadas en el litoral y empresas ubicadas en el interior de la península de Yucatán

\begin{tabular}{|r|r|r|}
\hline \multicolumn{1}{|r|}{ Turistas } & Litoral & \multicolumn{1}{|r|}{ Interior } \\
\hline & $(\mathrm{n}=88)$ & $(\mathrm{n}=65)$ \\
\hline & $\%$ & $\%$ \\
\hline $5,000-9,999$ & 55.7 & $76.9^{*}$ \\
\hline$\geq 10,000$ & 15.9 & 9.2 \\
\hline & 28.4 & 13.8 \\
\hline
\end{tabular}

$* \mathrm{p}<0.05$

Asimismo se encontró una asociación significativa y en el mismo sentido que la anterior, entre el total del ingreso bruto anual y la ubicación $\left(\mathrm{X}_{(2)}^{2}=31.919, \mathrm{p}=0.00000001\right)$. Las empresas ubicadas en el interior tienen ingresos significativamente menores que las ubicadas en el litoral (Cuadro 3).

Cuadro 3: Comparación del ingreso bruto anual entre empresas ubicadas en el litoral y empresas ubicadas en el interior de la península de Yucatán

\begin{tabular}{|r|r|r|}
\hline \multicolumn{1}{|c|}{ Ingreso } & Litoral & \multicolumn{1}{|r|}{ Interior } \\
\hline & $(\mathrm{n}=88)$ & $(\mathrm{n}=64)$ \\
\hline$<100,000$ & $\%$ & $\%$ \\
\hline $100,000-999,999$ & 14.1 & $32.8^{*}$ \\
\hline$\geq 1,000,000$ & 37.6 & 13.8 \\
\hline
\end{tabular}

$* \mathrm{p}<0.001$

La diferencia en los montos de financiamento recibidos por empresas ubicadas en el litoral y en el interior también fue estadísticamente significativa $\left(\mathrm{X}_{(2)}^{2}=8.585, \mathrm{p}=0.035\right)$. Los montos más altos se distribuyeron en empresas ubicadas en el interior (Cuadro 4).

Cuadro 4: Comparación del financiamiento total acumulado entre empresas ubicadas en el litoral y empresas ubicadas en el interior de la península de Yucatán

\begin{tabular}{|r|r|r|}
\hline \multicolumn{1}{|c|}{ Ingreso } & Litoral & \multicolumn{2}{|r|}{ Interior } \\
\hline & $(\mathrm{n}=88)$ & $(\mathrm{n}=64)$ \\
\hline & $\%$ & $\%$ \\
\hline $100,000-999,999$ & 67.0 & 50.8 \\
\hline$\geq 1,000,000$ & 25.0 & 24.6 \\
\hline
\end{tabular}

${ }^{*} \mathrm{p}<0.05$ 


\section{Heterogeneidad del litoral (litoral Caribe/litoral Yucatán y Campeche)}

Los resultados de las pruebas de $\mathrm{X}^{2}$ mostraron que la diferencia en el número de turistas anual recibido por empresas del litoral Caribe y por empresas del litoral Yucatán y Campeche no fue estadísticamente significativa $\left(\mathrm{X}_{(1)}^{2}=1.640, \mathrm{p}=0.200\right)$ (Cuadro 5).

En contraste, la diferencia en el ingreso bruto anual fue estadísticamente significativa $\left(\mathrm{X}_{(1)}^{2}=30.791\right.$, $p=0.00000003)$. El ingreso fue mayor en las empresas ubicadas en el litoral Caribe que en las ubicadas en el litoral Yucatán y Campeche (Cuadro 6).

Cuadro 5: Comparación del total anual de turistas entre empresas ubicadas en el litoral Caribe y empresas ubicadas en el litoral Yucatán y Campeche

\begin{tabular}{|r|r|r|r|}
\hline \multicolumn{1}{|r|}{ Ingreso } & Caribe & \multicolumn{2}{|r|}{ Yuc-Cam } \\
\hline & $(\mathrm{n}=46)$ & $(\mathrm{n}=42)$ \\
\hline & & $\%$ & $\%$ \\
\hline$>3,960$ & 43.5 & 57.1 \\
\hline & 56.90 & 56.5 & 42.9 \\
\hline
\end{tabular}

Cuadro 6: Comparación del ingreso bruto anual entre empresas ubicadas en el litoral Caribe y empresas ubicadas en el litoral Yucatán y Campeche

\begin{tabular}{|r|r|r|}
\hline \multicolumn{1}{|c|}{ Ingreso } & Caribe & Yuc-Cam \\
\hline & $(\mathrm{n}=46)$ & $(\mathrm{n}=42)$ \\
\hline & $\%$ & $\%$ \\
\hline$>1,010,000$ & 21.7 & 81.0 \\
\hline
\end{tabular}

$* \mathrm{p}<0.0001$

Los montos de financiamiento entre ubicaciones tampoco fueron significativamente diferentes $\left(\mathrm{X}_{(1)}^{2}\right.$ $=0.182, \mathrm{p}=0.669)($ Cuadro 7$)$.

Cuadro7: Comparación del financiamiento total acumulado entre empresas ubicadas en el litoral Caribe y empresas ubicadas en el litoral Yucatán y Campeche

\begin{tabular}{|r|r|r|}
\hline \multicolumn{1}{|c|}{ Financiamiento } & Caribe & \multicolumn{2}{|r|}{ Yuc-Cam } \\
\hline & $(\mathrm{n}=46)$ & $(\mathrm{n}=42)$ \\
\hline & $\%$ & $\%$ \\
\hline$>137,500$ & 47.8 & 52.4 \\
\hline & 52.2 & 47.6 \\
\hline
\end{tabular}

\section{Traspaís como área emergente (traspais/resto del interior)}

Los resultados de la comparación entre empresas ubicadas en el traspaís y el resto del interior de la península mostraron dos diferencias estadísticamente significativas. El número de turistas $\left(\mathrm{X}^{2}{ }_{(1)}\right.$ $=6.584, \mathrm{p}=0.010)$ y el ingreso bruto anual $\left(\mathrm{X}_{(1)}^{2}=8.741, \mathrm{p}=0.003\right)$ fueron mayores en las empresas ubicadas en el traspaís que en las ubicadas en el interior del resto de la península (Cuadros 8 y 9). 
Cuadro 8: Comparación del total anual de turistas entre empresas ubicadas en el traspaís y empresas ubicadas en el resto del interior

\begin{tabular}{|r|r|r|}
\hline \multicolumn{1}{|c|}{ Turistas } & Traspaís & No Traspaís \\
\hline & $(\mathrm{n}=19)$ & $(\mathrm{n}=51)$ \\
\hline & $\%$ & $\%$ \\
\hline$>1,200$ & 26.3 & 60.8 \\
\hline & 73.7 & $39.2^{*}$ \\
\hline
\end{tabular}

$* p=0.01$

Cuadro 9: Comparación del ingreso bruto anual entre empresas ubicadas en el traspaís y empresas ubicadas en el resto del interior

\begin{tabular}{|c|c|c|}
\hline Ingreso & Traspaís & No Traspaís \\
\hline & $(\mathrm{n}=19)$ & $(\mathrm{n}=51)$ \\
\hline & $\%$ & $\%$ \\
\hline$\leq 105,500$ & 21.1 & 60.8 \\
\hline$>105,500$ & 78.9 & $39.2^{*}$ \\
\hline
\end{tabular}

$* \mathrm{p}<0.005$

El monto del financiamiento no fue significativamente diferente entre las dos ubicaciones $\left(\mathrm{X}_{(1)}^{2}=\right.$ 0.072, $\mathrm{p}=0.788$ ) (Cuadro 10).

\section{Cuadro 10: Comparación del financiamiento total acumulado entre empresas ubicadas en el traspaís y empresas ubicadas en el resto del interior}

\begin{tabular}{|r|r|r|}
\hline \multicolumn{1}{|c|}{ Financiamiento } & Traspaís & \multicolumn{2}{|c|}{ No Traspaís } \\
\hline & $(\mathrm{n}=19)$ & $(\mathrm{n}=51)$ \\
\hline & $\%$ & $\%$ \\
\hline$>446,000$ & 52.6 & 49.0 \\
\hline$>446,000$ & 47.4 & 51.0 \\
\hline
\end{tabular}

\section{Discusión}

En este trabajo analizamos estadísticamente el comportamiento de dos variables relacionadas con el desempeño económico de las empresas, total anual de turistas e ingreso bruto anual, y de una variable relacionada con la política de impulso a este sector, financiamiento total acumulado, mostrando la relación que guardan entre sí esas tres variables, así como la relación entre cada variable y su ubicación clasificada de tres formas diferentes, con base en los resultados del Atlas (García de Fuentes et al., 2015): litoral e interior, costa Caribe y costa Yucatán-Campeche y traspaís e interior del resto de la península.

Los resultados mostraron una relación estadísticamente significativa entre turistas e ingresos, pero no de ambos con el financiamiento. Las empresas que reciben más turistas obtienen más ingresos, pero no han recibido más financiamiento. No obstante que la relación no alcanzó significancia estadística, su signo fue negativo. Es decir, pareciera que montos altos de financiamiento no se traducen en mejor desempeño económico, medido por el número de turistas y los ingresos. Estos resultados son congruentes con el conjunto de información del 
Atlas que sugiere que el financiamiento no garantiza que las empresas se desarrollen exitosamente en términos de su desempeño económico y que en los fracasos, si bien puede haber diversas causas, la localización juega un papel muy importante. Si bien se observa una diferencia con López G. y Palomino (2008) que concluyen que el financiamiento a fondo perdido (subsidio) es uno de los factores que contribuye al éxito de las empresas indígenas de turismo, existen coincidencias pues también consideran factores asociados a la localización (vías de acceso e integración a rutas turísticas o a paquetes estructurados), además de otros factores como las formas de organización colectiva (López, G. y Palomino 2015; Palomino et al., 2016).

Las empresas que captaron menos turistas y obtuvieron menos ingresos se ubican en el interior y fueron estas mismas empresas las que recibieron los montos más altos de financiamiento. En otras palabras, las empresas que muestran mejor desempeño económico se localizan en el litoral y han recibido montos menores de financiamiento. El relativo éxito de las empresas ubicadas en el litoral puede atribuirse a diversos factores, pero el principal es su localización. La mayoría de éstas surgieron de manera espontánea como respuesta de la población local, generalmente de pescadores, a una demanda generada por el turismo de masas, principalmente en el litoral del Caribe pero también en la costa norte de Yucatán. En cambio en el interior, el desarrollo de las empresas del sector social se relaciona más directamente con políticas, nacionales e internacionales, de fomento y financiamiento a la actividad, que ven en el turismo alternativo una opción factible frente al deterioro de la agricultura tradicional, que cada vez cuenta con menor apoyo pues su productividad no responde a los parámetros esperados en una economía comercial de alto rendimiento. Al mismo tiempo, el turismo alternativo es considerado un aliado de la conservación de los recursos naturales y ecosistemas, particularmente en el caso de las áreas naturales protegidas. A pesar de tratarse de una política pública para el desarrollo de indígenas y campesinos y coadyuvante de la conservación, el monto total del financiamiento otorgado a las empresas en la península entre 1994 y 2012 fue de $\$ 143.7$ millones de pesos (García de Fuentes et al., 2015), cantidad que resulta mínima para abarcar una región de la magnitud de la península; tan solo en la Riviera Maya, la inversión en un hotel puede ascender a más del doble de aquel monto. En tanto que en la mayoría de los casos se trata de financiamientos a fondo perdido, constituyen un subsidio (por ejemplo, ver CONEVAL s/f) con el que se pretende paliar las dificultades que enfrenta la agricultura tradicional y que son otorgados sin considerar la ubicación en términos del potencial de mercado.

Si bien las empresas ubicadas en el litoral muestran un mejor desempeño económico que las ubicadas en el interior, no todas las ahí localizadas lo logran. El análisis estadístico mostró diferencias entre las empresas del litoral del Caribe, donde domina el turismo masivo tanto internacional como nacional, y las del resto del litoral, cuyo mercado es predominantemente local. Las primeras obtienen ingresos más altos que las segundas, no obstante que ambas reciben un número similar de turistas. Estos resultados muestran que la oferta de turismo alternativo ofrecido por empresas del sector social, que por su ubicación se asocian con el turismo masivo, obtienen ingresos superiores. Por tanto, la opción real para que pescadores o campesinos basen su permanencia en el lugar a partir del turismo está relacionada con el acceso a ese turismo masivo internacional o nacional de ingresos medios y altos. En el caso del financiamiento no hubo diferencias significativas en los montos otorgados a empresas ubicadas en el litoral Caribe y las ubicadas en el litoral de Yucatán y Campeche. Nuevamente, estos resultados muestran que el otorgamiento de financiamiento no atiende a criterios relacionados con la ubicación.

La heterogeneidad se da también en los territorios del interior. En este caso, la diferencia más importante planteada en el Atlas se relaciona con una región turística emergente a la que se denomina traspaís del litoral Cancún-Riviera Maya. El análisis estadístico mostró que el número de turistas y el ingreso son mayores en las empresas ubicadas en el traspaís que las ubicadas en el interior del resto de la Península. Las empresas del traspaís se localizan en un triángulo con base en el litoral Cancún-Riviera Maya y vórtice en Chichén Itzá, que posibilita la conexión con touroperadores que ofrecen recorridos complementarios desde los principales centros de turismo masivo de sol y playa (Cancún, Playa del Carmen y Tulum), que aprovechan la cercanía a la oferta de empresas del sector social para vender la experiencia de adentrarse en la naturaleza "tropical", "salvaje" y "el mundo maya profundo". Mérida crea también un área de influencia en su entorno, relacionada principalmente con la oferta de cenotes, que no ha logrado competir con lo que ofrece el traspaís. Menor aún es la competencia que ofrecen un conjunto de empresas ubicadas en zonas más alejadas, relacionadas sólo con visitantes locales de fin de semana o, por ejemplo Calakmul al sur del estado de Campeche, con una afluencia mínima por su lejanía del mercado. Con relación al financiamiento, no se encontró diferencia en los montos recibidos por empresas del traspaís y fuera del traspaís. Nuevamente, se muestra la carencia de un enfoque territorial en la política de fomento del turismo alternativo hacia empresas del sector social. 


\section{Conclusiones}

La política pública de financiamiento a los campesinos para reconvertir su actividad hacia el turismo hace abstracción del territorio, con lo que su impacto, como confirma el trabajo, es muy pobre, y funciona en la mayoría de los casos, como un subsidio paliativo a situaciones de pobreza y abandono histórico, sin buscar realmente resultados positivos en la operación de los proyectos. Los resultados económicos de los emprendimientos se relacionan con su proximidad a los mercados masivos de turismo internacional y nacional, más allá de haber recibido apoyos financieros para iniciar o mantener la actividad.

Las relaciones observadas entre número de turistas e ingresos sugieren que existe una contradicción con los presupuestos del turismo alternativo como un turismo no masivo, ya que la viabilidad económica de estas empresas requiere de una relativa masificación pues su rentabilidad depende de números altos de turistas. Los casos de mayor éxito en la Península se localizan en el traspaís: Pacchén y Tres Reyes en el municipio de Lázaro Cárdenas, Q. Roo; Muyil en el municipio de Tulum, Q. Roo, y Zaaz Koolen Haá en la localidad de Yokdzonot del municipio de Yaxcaba, Yucatán. Estas empresas están recibiendo flujos de alrededor de 50000 turistas al año lo que significa afluencias diarias de más de 1000 turistas en los periodos altos (Lemas, 2015, Jimenez, 2012). En el caso de Zaaz Koolen Haá, los propios socios se están planteando que, en los días de gran afluencia, la visita ya no resulta placentera para los turistas (información recabada en campo, julio de 2018). Esto sugiere la necesidad de realizar estudios que analicen la manera de alcanzar un número suficiente de turistas que garanticen la rentabilidad de la empresa a largo plazo, estableciendo mecanismos de operación que mantengan la calidad del disfrute, basado en experiencias alternativas a las que brinda el turismo masivo.

Es necesario reconocer que los resultados aquí presentados se derivan de un análisis secundario; es decir, se utilizaron datos que fueron recogidos para elaborar el Atlas de turismo alternativo en la península de Yucatán y, por tanto, orientados a un análisis espacial. Debido al uso de la base de datos del Atlas, una limitante importante del presente trabajo es la dificultad en la obtención y el grado de precisión de la información sobre el total anual de turistas y el ingreso bruto anual ya que las empresas del sector social, en su inmensa mayoría, no cuentan con el conocimiento necesario para llevar un registro administrativo de corte empresarial. En todo caso, al seguir un mismo procedimiento para obtener la información necesaria para estimar esas dos variables, como se señala en la Metodología, de haber alguna subestimación o sobreestimación, ésta sería similar en todos los casos. Es decir, el sesgo sería similar. Debido a la naturaleza de los datos, el análisis estadístico se vio limitado al establecimiento de asociaciones univariadas. Se exploraron transformaciones de datos de diversa índole; sin embargo, los resultados no fueron satisfactorios.

Los resultados muestran que, si bien los ingresos dependen del número de turistas, la ubicación puede ser más relevante pues puede modificar esta asociación. La relación con el turismo masivo juega un papel fundamental como se muestra en el caso del traspaís y también en el caso del litoral Caribe. La política de fomento al turismo alternativo como opción frente a la agricultura tradicional, considerada como no viable bajo el esquema de altos rendimientos y comercialización (por ejemplo, Bartra y Otero, 1987; de Frece y Poole, 2008; García-Barro y García-Barro, 1990), parece basarse en la idea de que todos los lugares son susceptibles de convertirse en producto turístico sin considerar la heterogeneidad de esos lugares. No solo litoral e interior son distintos sino también el litoral y el interior son heterogéneos y esta heterogeneidad en la localización también parece ser ignorada por la política de fomento al turismo alternativo del sector social de la economía.

Como señala Vera et al. (1997: 51) “... el turismo es un fenómeno espacial, hecho que con frecuencia se minimiza o se obvia, debido al predominio de la concepción y análisis económico del mismo”. El turismo es una de las actividades que más consumo de espacio o más impacto por su expansión en el territorio tiene en la actualidad. El turismo va más allá de la actividad económica en sí (pago por el disfrute de recursos naturales y culturales); es al mismo tiempo una respuesta a la necesidad de las poblaciones urbanas de volver a la naturaleza, una respuesta a la necesidad posmoderna de reconocer y revalorar al otro (en este caso otras culturas). Es una forma de vida ligada al uso del tiempo libre, cada vez más masiva. Y, en estos sentidos, es una actividad que genera un consumo brutal de espacio y que, desde el punto de vista económico, es idónea para el negocio de la especulación con la tierra a mediano y largo plazo. En este sentido, los resultados encontrados abren interrogantes sobre el objetivo de impulsar al turismo alternativo en cualquier lugar, de manera indiscriminada, sin importar los resultados que se obtengan, lo que podría relacionarse con las consideraciones de Fletcher (2010), López, A. (2015), López, A., y Marín (2012) de que este tipo de turismo es una forma sofisticada del capital para apropiarse de territorios biodiversos y con un capital cultural que, generalmente, se encuentran en manos de los 
pueblos indígenas, y con lo citado de Urry (2001) sobre la manera en que se reconfiguran cada vez más lugares como sitios de producción y consumo global del turismo.

\section{Bibliografía}

Armenta, G. 15 de marzo de 2019. Ciudad abierta. Xcaret refrenda sus inversiones en México. El Financiero, consultado en https://elfinanciero.com.mx/opinion/gustavo-armenta/xcaret-refrenda-sus-inversiones-en-mexico.

Barragán, J. A. 2015. Prácticas ambientales de los emprendimientos ecoturísticos en la costa de Yucatán: Un estudio regional. Tesis de Maestría en Ciencias en la especialidad de Ecología Humana. Centro de Investigación y de Estudios Avanzados, Unidad Mérida). Recuperado de https://www.mda.cinvestav. $\mathrm{mx} / \mathrm{FTP} /$ EcologiaHumana/maestria/tesis/12TesisBarraganA15.pdf.

Bartra, R., \& Otero, G. 1987. Agrarian crisis and social differentiation in Mexico. The Journal of Peasant Studies, 14(3), 334-362.

Bringas, N., y Ojeda, L. 2000. El ecoturismo: ¿una nueva modalidad del turismo de masas? Economía, Sociedad y Territorio, 2, 373-403.

Ceballos, H. 1998. Ecoturismo, naturaleza y desarrollo. México: Editorial Diana.

CONEVAL s/f. Informe de la evaluación específica de desempeño 2012-2013. Valoración de la información de desempeño presentado por el programa. Documento disponible en http://www.cdi.gob.mx/coneval/ S184_PTAZI_EED_COMPLETO_2012_2013.PDF).

Coraggio, J. L. 2011. La sostenibilidad de los emprendimientos de la economía social y solidaria. Otra Economía, 2, 41-57.

Cheia, G. 2013. Ecotourism: definition and concepts. Revista de Turismo-Studii si Cercetari in Turism, $15,56-60$.

Cohen, J. 1990. Things I have learned (so far). American Psychologist 45, 1304-1312.

Daltabuit, M., Hernández, A., Barbosa, S., y Valdez, A. 2007. Reflexiones en torno al ecoturismo en Yucatán. Península, 2, 103-124.

de Frece, A. y Poole, N. 2008. Constructing livelihoods in rural Mexico: milpa in Mayan agriculture. The Journal of Peasant Studies, 35, 335-352.

Félix-Quezada, M. 2018. De campesinos indígenas a promotores de turismo. La experiencia del ejido San Cristóbal Hidalgo, México. Agricultura, Sociedad y Desarrollo, 15, 247-274.

Fletcher, R 2016. Tours caníbales puesto al día: la ecología política del turismo. Ecología Política. Cuadernos de Debate Internacional, 52, 26-34.

García-Barro, R. y García-Barro, L. 1990. Environmental and technological degradation in peasant agriculture: a consequence of development in Mexico. World Development, 18, 1569-1585.

García de Fuentes, A., Jouault, S., y Romero, D. 2015. Atlas de turismo alternativo de la Península de Yucatán. Mérida, México: Cinvestav-Mérida y Facultad de Ciencias Antropológicas, UADY.

Garduño, M., Guzmán, C., y Zizumbo, L. 2009. Turismo rural: participación de las comunidades y programas federales. El Periplo Sustentable, 17, 5-30.

Gasca, J., López, G., Palomino, B., y Mathus, M. 2010. La gestión comunitaria de recursos naturales y ecoturísticos en la Sierra Norte de Oaxaca. México, D.F.: IIE UNAM y Academia Mexicana de Investigaciones Turísticas.

Jiménez, M. 2016. Perspectivas de los jóvenes frente al turismo en el traspaís del corredor Cancún-Riviera Maya. Tesis de Maestría en Ciencias en la especialidad de Ecología Humana. Centro de investigación y de Estudios Avanzados, Unidad Mérida. Recuperado de https://www.mda.cinvestav. $\mathrm{mx} / \mathrm{FTP} /$ EcologiaHumana/maestria/tesis/13TesisJimenezM16.pdf.

Lemas, M. F. 2019. Empresas turísticas de la economía social y recursos de uso común. Estudio de tres cooperativas del traspaís de Cancún-Riviera Maya. Tesis de Maestría en Ciencias en la especialidad de Ecología Humana. Centro de investigación y de Estudios Avanzados, Unidad Mérida. Recuperado de https://www.mda.cinvestav.mx/FTP/EcologiaHumana/maestria/tesis/17LemasM19.pdf.

Jouault, S., García de Fuentes, A., y Rivera, T. 2015. Un modelo regional de turismo alternativo y economía social en la Península de Yucatán, México. Otra Economía, 9, 164-176.

López, A. 2015. Quemar las naves. El despojo articulado de la Isla de Holbox, en G. Marín (Ed.), Sin tierras no hay paraíso. Turismo, organizaciones agrarias y apropiación territorial en México (pp. 39-58). Colección Pasos, 15. Santa Cruz de Tenerife, Islas Canarias: Pasos 
López, A., y Marín, G. 2012. Ecoturismo, desarrollo y sustentabilidad: un recorrido por senderos interpretativos de poder, mercado y simulacro, en A. Castellanos, y J. Machuca (Eds.), Turismo y antropología: miradas del sur y el norte (pp. 201-232). México, D.F.: Juan Pablos.

López, G., y Palomino, B. 2008. Políticas públicas y ecoturismo en comunidades indígenas de México. Teoría y Praxis, 5, 33-50.

López, G., y Palomino, B. 2015. Situación general de las empresas indígenas de turismo de naturaleza en México. TEACS, 7, 93-106.

Marañón, B., y López D. 2013. Una propuesta teórico-metodológica crítica para el análisis de las experiencias populares colectivas de trabajo e ingresos: hacia una alternativa societal basada en la reciprocidad. En B. Marañón (Coord.), La economía solidaria en México (pp. 25-78). México, D.F.: UNAM.

OMT. 1995. Carta del turismo sostenible, Lanzarote, Islas Canarias, España. Conferencia Mundial de Turismo Sostenible. Organización Mundial de Turismo: Santa Cruz Tenerife, Islas Canarias.

OMT. 2001. Turismo: Panorama 2020. Vol 7. Previsiones mundiales y perfiles de los segmentos de mercado. World Tourism Organisation. Recuperado de https://www.e-unwto.org/doi/book/10.18111/9789284404766.

Palomino, B., Gasca, B. y López, G. 2016. El turismo comunitario en México: perspectiva desde las instituciones y la gobernanza en territorios indígenas. El Periplo Sustentable, 30, 6-37.

Pulido, M. E. 2014. Diagnóstico de la problemática administrativa en empresas de turismo alternativo a partir de tres estudios de caso en Yucatán. Tesis de Maestría en Ciencias en la especialidad de Ecología Humana. Centro de Investigación y de Estudios Avanzados, Unidad Mérida). Recuperado de https://www.mda.cinvestav.mx/FTP/EcologiaHumana/maestria/tesis/11TesisPulidoE14.pdf.

Salciccia, D. 2001. El ecoturismo rural y el desarrollo sustentable del patrimonio natural e histórico cultural. Estudios y Perspectivas en Turismo, 10, 3-15.

Urry, J. 2002. The tourist gaze: leisure and travel in contemporary societies. Londres: Sage.

Warman, A. 2001. El campo mexicano en el siglo XX. México, D.F.: Fondo de Cultura Económica.

Vera, J. F., López, F., Marchena, M., y Antón, S. 1997. Análisis territorial del turismo: una nueva geografía del turismo. Barcelona, España: Ariel.

\section{Notes}

1 La península de Yucatán está integrada por los estados de Yucatán, Campeche y Quintana Roo que en conjunto abarcan una superficie de $140000 \mathrm{Km}^{2}$, el 7\% del país, y suman una población de 4.1 millones de habitantes, el 3.7\% de la población del país en 2015.

2 En adelante, utilizaremos localización y ubicación como sinónimos.

3 Es cierto que el capital, a partir de grandes inversiones, puede desarrollar el turismo en los lugares más recónditos, inaccesibles o inesperados.

4 1) Datos de identificación de la empresa; 2) datos sobre la historia de la empresa; 3) Situación legal del terreno donde opera el proyecto; 4) número y características de los campesinos participantes; 5) capacitación recibida; 6) vinculación con Áreas Naturales Protegidas; 7)actividades y servicios que ofertan; 8) actividades pro ambientales y culturales que realizan; 9) tipo de recursos naturales o culturales comercializados; 10) infraestructura con que cuentan; 11) número de visitantes (turistas) y su origen; 12) ingresos brutos anuales de la empresa; 13) vinculación con agencias de viajes y turoperadores; 14) egresos y salarios percibidos; 15) mecanismos de promoción; 16) financiamientos recibidos por fuente y año; 17) principales problemas enfrentados.

5 El primer año en que se otorgaron apoyos financieros a este tipo de turismo fue 1994, aunque las primeras empresas iniciaron en la década de los 70 s.

6 Por ejemplo, el grupo Xperiencias Xcaret invirtió alrededor de 330 millones de dólares en el hotel Xcaret México entre 2016 y 2017 (Armenta, 2019). 